\title{
Long-Term Outcomes after D2 Gastrectomy for Early Gastric Cancer: Survival Analysis of a Single-Center Experience in China
}

\author{
Zheng Wang ${ }^{1 \&}$, Li Ma ${ }^{2 \&}$, Xing-Mao Zhang ${ }^{1}$, Zhi-Xiang Zhou ${ }^{1 *}$
}

\begin{abstract}
Background: Early gastric cancer (EGC) is well accepted as having a favorable prognosis, but some patients experience an ominous outcome after curative resection. This study was aimed at evaluating predictive factors associated with prognosis of $\mathrm{D} 2$ gastrectomies in patients with early gastric cancer. Materials and Methods: A total of 518 patients with early gastric cancer who underwent D2 gastrectomies were reviewed in this study. The clinicopathological features and surgical outcomes were analyzed. The survival rate was estimated using the Kaplan-Meier method and compared by log rank test. Prognostic factors were analyzed using a multivariate Cox proportional hazards model. Results: The 5-year survival rate was $\mathbf{9 0 . 3 \%}$. Tumor infiltration, lymph node metastasis and lymphovascular invasion were significant prognostic factors for survival. Gender, age, tumor size, tumor location, macroscopic type and histological type were not significant prognostic factors. Multivariate analysis indicated that lymph node metastasis was an independent poor prognosis factor. Conclusions: Early gastric cancers with lymph node metastasis have a relatively poor prognosis after standard surgery. Even after curative resection, patients with EGC with positive lymph nodes should be closely followed and be considered as candidates for comprehensive therapies.
\end{abstract}

Keywords: Early gastric cancer - prognostic factor - lymph node metastasis - surgery

Asian Pac J Cancer Prev, 15 (17), 7219-7222

\section{Introduction}

In 1962, the Japanese Society of Gastroenterological Endoscopy defined early gastric cancer as a lesion confined to the mucosa and/or submucosa, regardless of lymph node metastatic status (Murakami et al., 1971). The prognosis is generally favorable in early gastric cancers after radical surgery, with a 5-year survival rate of more than $90 \%$ (Hyung et al., 2002; Kwee et al., 2008). With more widespread mass screenings, as well as advances in endoscopic techniques and equipment, more early gastric cancer patients have been observed during the past few decades in China, and the frequency of early stage has grown steadily among patients who underwent resections for gastric cancers.

Many investigations of early gastric cancer have shown that the existence of lymph node metastasis decreases survival. Therefore, lymph node metastasis is regarded as having a significant influence on prognosis and on the selection of possible treatment (Ohta et al., 1987; Haves et al., 1996; Hyung et al., 2002; Kwee et al., 2008; Tatematsu et al., 2013). D2 gastrectomy has been routinely employed in patients with early gastric cancers, and excellent curative treatment has been obtained with regional lymphadenectomy. Recently, less invasive treatments have been performed for early gastric cancer, including endoscopic mucosal resection (EMR), endoscopic submucosal dissection (ESD) and limited lymphadenectomy.

The present study was designed to analyze the clinicopathological characteristics of early gastric cancer in a large group of patients from China and to characterize the factors that influence the 5-year survival rate in patients undergoing radical resection for early gastric cancer.

\section{Materials and Methods}

\section{Patients}

Institutional review board approval was obtained before conducting this study. From January 1994 to December 2008, 4, 586 consecutive patients with gastric cancer underwent curative gastrectomies with D2 lymph node dissection at the Department of Abdominal Surgical Oncology, Cancer Hospital of the Chinese Academy of Medical Sciences, Peking Union Medical College, Beijing. Among those patients, 518 (11.3\%) patients with complete follow-up inforemation who underwent surgery and achieved radical (R0) resection for histologically

${ }^{1}$ Department of Abdominal Surgical Oncology, Cancer Hospital of the Chinese Academy of Medical Sciences, Peking Union Medical College, ${ }^{2}$ Department of Respiration, First Affiliated Hospital of PLA General Hospital, Beijing, China \&Equal contributors *For correspondence: zhouzx2014@gmail.com 
proven primary early gastric cancer were retrospectively analyzed in this study. Cases with recurrence or multifocal gastric cancer were excluded. No patient has received neoadjuvant therapy before surgery.

All patients underwent radical subtotal or total gastrectomies, depending on the tumor location and intraoperative verification of tumor-free resection margins, as well as D2 lymphadenectomies. The methods of reconstruction after distal gastrectomy include Billroth I, II or Roux-en-Y. Roux-en-Y reconstruction and jejunal interposition are the most common methods used after a total gastrectomy.

The patients' clinical characteristics, histopathological parameters and follow-up data were analyzed. Clinicopathological factors, such as gender, age, tumor size, tumor location, macroscopic appearance, histological type, depth of tumor invasion, lymph node status and lymphovascular invasion, were defined according to the General Rules of the Japanese Classification of Gastric Carcinoma ( $2^{\text {nd }}$ English edition) (Japanese Gastric Cancer Association Association, 1998). Macroscopic type includes elevated (I, protruded; IIa, superficial elevated), flat (IIb, superficial flat), depressed (IIc, superficial depressed; III, excavated) and mixed (depressed plus elevated). Tumor histology was classified into two groups: 1) differentiated, which included papillary adenocarcinoma and well or moderately differentiated adenocarcinoma; and 2) undifferentiated, which included poorly or undifferentiated adenocarcinoma, signet ring cell carcinoma and mucinous carcinoma. The depth of tumor invasion was classified as mucosa and submucosa carcinoma. Lymphovascular invasion was defined as the presence of tumor emboli either in the lymphatic duct or the vascular lumen.

Patients were examined every three months for the first two years and every six months thereafter. The followup protocol involved standardized clinical examinations, including gastroscopy, tumor markers, chest $\mathrm{x}$-ray and abdominal CT scans. All patients were followed-up until death or the cutoff date (May 31, 2014). The median follow-up time was 99 months (range, 65-238). Only deaths due to early gastric cancer were considered. Deaths due to other causes were censored at the date of death.

\section{Statistical analysis}

Survival data for the 518 patients were analyzed using the Kaplan-Meier method. The log rank test was used to assess the differences between prognostic factors. A multivariate analysis of the factors influencing the 5-year survival rate was performed using the Cox proportional hazards model. $\mathrm{P}<0.05$ was considered statistically significant. Statistical analyses were performed with SPSS software, version 15.0 for Windows (SPSS Inc., Chicago, IL, USA).

\section{Results}

The study involved 349 men and 169 women, with a male:female ratio of $2.07: 1$. The median age of the enrolled patients was 58 years (24-82 years). Of the 518 patients, metastatic lymph node involvement was present in $79(15.3 \%)$. The mucosal form of cancer presented with lymph node involvement in $3.3 \%$ of cases, in contrast to $23.5 \%$ of cases that were associated with submucosal forms. Tumor invasion was limited to the mucosal layer in 212 patients $(40.9 \%)$, while in 306 patients $(59.1 \%)$, the tumors had invaded the submucosal layer. A total of 375 patients had tumors located in the lower third of the stomach. Eighty-four had tumors in the middle third of the stomach, while 59 had tumors in the upper third. Depressed-type tumors were macroscopically observed in 273 patients, flat-type tumors were observed in 188 patients, and elevated-type or mixed-type tumors were observed in the remaining 57 patients. The majority of patients had a tumor diameter greater than $2.0 \mathrm{~cm}$. Nearly two-thirds of the identified tumors were well differentiated.

Data for the survival analysis were available for all patients who underwent operations in December 2008 or earlier. The 5-year survival rate was $90.3 \%$. Factors associated with survival, as determined by KaplanMeier and log rank analyses, are presented in Table 1. The univariate analysis revealed that there was no significant impact of gender, age, tumor size, tumor location, macroscopic type or histological type. The factors associated with poor survival were tumor invasion, lymph node metastasis and lymphovascular invasion. The multivariate Cox proportional regression analysis showed that lymph node metastasis was an independent risk factor (96.2\% versus $64.3 \%, p<0.01$, Table 2 ).

Table 1. Univariate Analysis of Factors Related to the Prognosis of Early Gastric Cancer

\begin{tabular}{|c|c|c|c|c|}
\hline \multicolumn{2}{|l|}{ Factor } & \multicolumn{3}{|c|}{$\begin{array}{l}\mathrm{n} \quad \text { 5-year } p \text { value } \\
\text { survival rate }(\%)\end{array}$} \\
\hline \multirow[t]{2}{*}{ Gender } & Male & 349 & 90.3 & 0.92 \\
\hline & Female & 169 & 90.4 & \\
\hline \multirow[t]{2}{*}{ Age (years) } & $\leq 60$ & 336 & 91 & 0.89 \\
\hline & $>60$ & 182 & 89.2 & \\
\hline \multirow[t]{3}{*}{ Tumor Size (cm) } & $<1.0$ & 68 & 88.4 & 0.85 \\
\hline & $1.0-2.0$ & 174 & 86.5 & \\
\hline & $>2.0$ & 276 & 92.5 & \\
\hline \multirow[t]{3}{*}{ Tumor Location } & Upper & 59 & 89.5 & 0.87 \\
\hline & Middle & 84 & 90.6 & \\
\hline & Lower & 375 & 90.4 & \\
\hline \multirow[t]{4}{*}{ Macroscopic Type } & Elevated & 39 & 90.5 & 0.93 \\
\hline & Flat & 188 & 89.3 & \\
\hline & Depressed & 273 & 91 & \\
\hline & Mixed & 18 & 88.5 & \\
\hline \multirow[t]{2}{*}{ Histological Type } & Differentiated & 183 & 87.2 & 0.62 \\
\hline & Undifferentiated & 335 & 91.9 & \\
\hline \multirow[t]{2}{*}{ Depth of Invasion } & Mucosal & 212 & 95.7 & 0.02 \\
\hline & Submucosal & 306 & 84.8 & \\
\hline \multirow[t]{2}{*}{ Lymphovascular Invasion } & Positive & 18 & 77.8 & 0.01 \\
\hline & Negative & 500 & 90.8 & \\
\hline \multirow[t]{2}{*}{ Lymph Node Metastasis } & Negative & 439 & 96.2 & 0.003 \\
\hline & Positive & 79 & 64.3 & \\
\hline
\end{tabular}

Table 2. Cox Multiple Regression Analysis of Early Gastric Cancer

\begin{tabular}{lcccc}
\hline Factor & $\begin{array}{c}\text { Regression } \\
\text { coefficient }\end{array}$ & $\begin{array}{c}\text { Standard } \\
\text { error }\end{array}$ & $\begin{array}{c}\text { Relative } \\
\text { risk }\end{array}$ & $p$ value \\
Lymph Node Metastasis & 1.659 & 0.645 & 6.156 & 0.005 \\
Lymphovascular Invasion & 0.709 & 0.512 & 2.007 & 0.19 \\
Submucosal Invasion & 0.837 & 0.524 & 1.997 & 0.21 \\
\hline
\end{tabular}




\section{Discussion}

Gastric carcinoma is the major cause of cancer related deaths in in asia (Baghestani et al., 2009; Lee et al., 2011), and gastrectomy with lymph node dissection is the only curative management option. In Japan, as many as 30-50\% of all diagnosed gastric cancers are early gastric cancers (Tatematsu et al., 2013). In our experience, the incidence of early gastric cancer has gradually increased with more widespread mass screenings and advances in diagnostic techniques and equipment. Early gastric cancer represents $11.3 \%$ of all resected gastric cancers. Increased detection of early gastric cancers has significantly improved therapeutic outcomes for these patients.

The prognosis of patients with early gastric cancer remains very favorable compared to that of advanced tumor patients. In Japan, the 5-year survival rate of early gastric cancer is reported to exceed $90 \%$. In general, our long-term results are similar to those published by Japanese authors; in our analysis of 518 patients who underwent D2 radical surgeries for early gastric cancer, the 5-year survival rate was $90.3 \%$.

This study demonstrates that submucosal infiltration, lymphovascular invasion and lymph node metastasis are associated with decreased long-term survival for early gastric cancer. Additionally, a multivariate analysis showed that lymph node metastasis is an independent prognostic factor. The presence of lymph node metastasis in early gastric cancer results in a worse prognosis, as reported by Kitamura et al. (1997) and Folli et al. (2001). Noh et al. (2005) reported that the 5-year survival rate of early gastric cancer without lymph node metastasis is $94.2 \%$ and that of early gastric cancer with lymph node metastasis is $87.3 \%$. Nio et al. (1993) reported a 5-year survival rate of $95 \%$ for $\mathrm{N} 1$ patients and $68.4 \%$ for $\mathrm{N} 2$ patients for early gastric cancers, and Miwa et al. (1984) observed similar rates of $90 \%$ and $79 \%$, respectively. Seto et al. (1997) reported a 5-year survival rate of $74 \%$ for patients with $>4$ positive lymph nodes. The prognosis of patients with early gastric cancers confined to the mucosa was also reported to be better than cases involving invasions into the submucosa.

The incidence of lymph node metastasis is usually reported to be 5.7-20\% for early gastric cancer in Japan and Western countries (Borie et al., 2000; Abe et al., 2005). This study found a $15.3 \%$ incidence of lymph node metastasis. The incidence of lymph node metastasis in early gastric carcinoma is low, but it is important to identify early gastric cancer patients who are at a higher risk of lymph node metastasis and could benefit from more extended lymphadenectomy.

Gastrectomy with lymph node dissection is the most widespread surgical procedure used to treat early gastric cancers worldwide. Many studies have reported significant benefits following radical lymphadenectomy in early gastric cancers. Furthermore, gastrectomy with D2 lymphadenectomy can be performed with a low complication rate and was found to significantly improve the 5-year survival rate. Siewert et al. (1996) claimed that D2 lymphadenectomy could improve prognosis in patients without lymph node metastases. The reason for this improvement is that the micrometastases found in approximately $10 \%$ of the cases can only be detected with immunohistochemical techniques that are not routinely used. Thus, a small portion of patients with early gastric cancers might benefit from D2 lymph node dissection.

Because long-term survival after gastrectomy is excellent, issues surrounding quality of life after surgical treatment are becoming increasingly important. Considering the lower incidence of lymph node metastasis, limited surgery for selected patients has been suggested to improve the quality of life. Minimally invasive treatments, including endoscopic mucosal resection (EMR), endoscopic submucosal dissection (ESD) and limited lymphadenectomy, are associated with surgical results that are no worse than those of conventional surgery (Ono et al., 2001). Endoscopic resection is widely performed as an alternative treatment to surgery in patients with mucosal tumors smaller than $20 \mathrm{~mm}$ that are of histologically differentiated types without ulceration, which are estimated to carry no risk of lymph node metastasis (Gotoda et al., 2006). In contrast to open procedures, EMR, ESD and limited lymphadenectomy have been performed in recent studies with nearly zero complications. Recently, because of advances in surgical instrumentation and techniques, laparoscopic procedures have been suggested as an alternative treatment modality for early gastric cancer. Compared to conventional open gastrectomy, laparoscopic gastrectomy has several clinical advantages, including less pain, less inflammatory response, faster recovery of the gut function, shorter hospital stay, and better quality of life (Adachi et al., 2002).

On the basis of our results, we conclude that: (1) Radical D2 gastrectomy is the standard treatment for those patients with risk factors associated with lymph node metastasis in early gastric cancer; (2) early gastric cancer with lymph node metastasis has a relatively poor prognosis after standard surgery, so these patients should accept comprehensively adjuvant treatment and strict followup evaluations. It will be important to perform further evaluations in a larger population to confirm these results.

\section{References}

Abe N, Mori T, Takeuchi H, et al (2005). Laparoscopic lymph node dissection after endoscopic submucosal dissection: a novel and minimally invasive approach to treating early gastric cancer. Am J Surg, 190, 496-503.

Adachi Y, Shiraishi N, Kitano S (2002). Modern treatment of early gastric cancer: review of the Japanese experience. Dig Surg, 19, 333-9.

Baghestani AR, Hajizadeh E, Fatemi SR (2009). Bayesian analysis for survival of patients with gastric cancer in Iran. Asian Pac J Cancer Prev, 10, 823-6.

Borie F, Millat B, Fingerhut A, et al (2000). Lymphatic involvement in early gastric cancer. Arch Surg, 135, 121823.

Folli S, Dente M, Dell Amore D, et al (1995). Early gastric cancer: prognostic factor in 223 patients. Br J Surg, 82, 952-6.

Gotoda T, Yamamoto H, Soetikno RM (2006). Endoscopic submucosal dissection of early gastric cancer.J Gastroenterol, 41, 929-42. 


\section{Zheng Wang et al}

Haves N, Karat D, Scott DJ, et al (2002). Radical lymphadenectomy in the management of early gastric cancer. Br J Surg, 83, 1421-3.

Hyung WJ, Noh SH, Lee JH, et al (2002). Early gastric carcinoma with signet ring cell histology. Cancer, 94, 78-83.

Japanese Gastric Cancer Association (1998). Japanese classification of gastric carcinoma- $2^{\text {nd }}$ English edition. Gastric Cancer, 1, 10-24.

Kitamura K, Yamaguchi T, Taniguchi M, et al (1997). Analysis of lymph node metastasis in early gastric cancer: rationale of limited surgery. J Surg Oncol, 64, 42- 7.

Kwee RM, Kwee TC (2008). Predicting lymph node status in early gastric cancer. Gastric Cancer, 11, 134-48.

Lee YY, Oh DK, Choi KS, et al (2011). The current status of gastric cancer screening in Korea: report on the National Cancer Screening Programme, 2009. Asian Pac J Cancer Prev, 12, 3495-500.

Miwa K (1984). Evaluation of the TNM classification of stomach cancer and proposal for its rationale stagegrouping. Jpn J Clin Oncol, 14, 385- 410.

Murakami T (1971). Pathomorphological diagnosis: definition and gross classification of early gastric cancer. Gann Monogr Cancer Res, 11, 53-5.

Nio Y, Tsubono M, Kawabata K, et al (1993). Comparison of surgical curves of gastric cancer patients after surgery according to the UICC stage classification and the general rules for gastric cancer study by the Japanese Research Society for Gastric Cancer. Ann Surg, 218, 47- 53.

Noh SH, Hyung WJ, Cheong JH (2005). Minimally invasive treatment for gastric cancer: approaches and selection process. J Surg Oncol, 90, 188-93.

Ohta H, Noguchi Y,Takagi K, et al (1987). Early gastric carcinoma with special reference to macroscopic classification. Cancer, 60, 1099-106.

Ono H, Kondo H, Gotoda T, et al (2001). Endoscopic mucosal resection for treatment of early gastric cancer. Gut, 48, 222-9.

Seto Y, Nagawa H, Muto T (1997). Impact of lymph node metastasis on survival with early gastric cancer. World $J$ Surg, 21, 186-90.

Siewert R, Kestlmeier R, Bush R, et al (1996). Benefits of D2 lymph node dissection for patients with gastric cancer and pN0 and pN1 lymph node metastases. Br J Surg, 83, 1144-7.

Tatematsu H, Miyahara R, Shimoyama Y, et al (2013). Correlation between magnifying narrow-band imaging endoscopy results and organoid differentiation indicated by cancer cell differentiation and its distribution in depressedtype early gastric carcinoma. Asian Pac J Cancer Prev, 14, 2765-9. 